\title{
Actividades Cocurriculares en Elena de White y la Propuesta Educativa en un Mundo Posmoderno
}

\author{
Angel Becerra Santa Cruz ${ }^{1 \mathrm{a}}$ \\ Universidad Peruana Unión ${ }^{1}$
}

Orcid ID: https://orcid.org/0000-0003-4053-92571

\section{Recibido: 21 de noviembre de 2017}

Aceptado: 05 enero de 2018

\section{Resumen}

En el contexto socioeconómico de su época, Elena de White llamó a la agricultura el ABC del currículum para recalcar su importancia en la educación de una iglesia, además de actividades como la culinaria, confección, colportaje, entre otras. Todas ellas como acciones co-curriculares en contribución al desarrollo armonioso de las facultades humanas de las que los pensamientos modernos no necesariamente los contemplan. A partir de una investigación exploratoria pretendemos comparar las propuestas de las actividades co-curriculares en Elena White con la de autores contemporáneos. Los resultados indican que las actividades cocurriculares en Elena White, trasciende las propuestas contemporáneas porque prepara a los estudiantes física, social, espiritual e intelectualmente; en tanto que la propuesta contemporánea considera que las actividades cocurriculares son un apoyo fundamental para los estudiantes y sirven para ampliar sus niveles de conocimiento, investigación y currículo.

Palabras clave: Actividades cocurriculares, propuestas educativas, mundo posmoderno.

\section{Co-curricular Activities in Ellen White and the proposal of Education in a Postmodern}

\section{World}

\begin{abstract}
In the socioeconomic context of her time, Ellen White called agriculture the $\mathrm{ABC}$ of the curriculum to emphasize its importance in the education of a church, in addition to activities such as cooking, baking, canning, and colportering, among others. All of these are co-curricular activities which contribut to the harmonious development of the human faculties which are not necessarily taken into account in modern thought. Based in an exploratory research study we intend to compare the proposals of the co-curricular activities in Ellen White with that of contemporary authors. The results indicate that the co-curricular activities in Ellen White transcend contemporary proposals because they prepare the students physically, socially,
\end{abstract}


spiritually and intellectually, while the contemporary proposal considers that the co-curricular activities are afundamental support for the students and serve to expand their levels of knowledge, research and curriculum.

Key-words: Co-curricular activities, educational proposals, post-modern world

\section{Introducción}

La mayoría de las instituciones educativas proporcionan prioritariamente instrucción académica; existiendo otras pocas que participan en la formación integral de los niños y jóvenes a través de sus programas académicos y de las actividades extra-académicas. Castro (2003) realizó una investigación que se interesa por conocer el papel que juega la formación cocurricular en el incremento de la calidad académica de los estudiantes de la licenciatura en ciencias de la educación de la UJAT, de la Universidad Juárez Autónoma de Tabasco, México, cuyo objetivo fue exponer las actividades cocurriculares o extracurriculares y su incidencia en la formación de los estudiantes de la licenciatura en Ciencias de la Educación con una muestra de 107 estudiantes, en el que llega a las siguientes conclusiones: 1. Las actividades cocurriculares son un apoyo fundamental para los estudiantes y sirven para ampliar sus niveles de conocimiento en calidad educativa, investigación y currículo. 2. Los alumnos consideran, que efectivamente las actividades cocurriculares o extracurriculares enriquecen sus conocimientos.

Por otro lado, Palacios (2012) realizó una investigación sobre las actividades extracurriculares y el mejoramiento cualitativo de la Educación, en la Universidad Estatal de Milagro, Ecuador, a través de una investigación descriptiva experimental, de tipo relacional buscó determinar y poner en práctica las actividades extracurriculares que tiene relación con la formación académica de los estudiantes. Concluye que: 1. se considera que una buena aplicación de técnicas y estrategias metodológicas aplicadas al proceso enseñanza - aprendizaje en las actividades extracurriculares, contribuirá a: A mejorar el desarrollo de las destrezas y habilidades de los estudiantes. A lograr estudiantes que tengan agrado por cualquier asignatura y evitar el memorismo tradicionalista. 2. La educación actual debe, perseguir el doble objetivo de ser productivos y ser formativos e incluir el aprendizaje de lo interhumano en una realidad social. 3. Si se practican las actividades extracurriculares con disciplina bien planificada, de seguro el estudiante va a continuar en el futuro con perseverancia para aprender nuevas cosas, entusiasmo, motivación y seguridad de sí mismo logrado el éxito deseado.

Se han realizado otras investigaciones y existen opiniones respecto a las actividades cocurriculares o llamadas por otros extracurriculares en las que se ha observado resultados 
favorables respecto a sus propósitos (Simôes \& Rodrigues, 2011). (Asociación de Profesores de Radiologia y Medicina Física, 2011) (Dabbah, 2007) (Plan Nacional de Evaluación de la calidad de las Universidades, 2003); Sin embargo, no existe una propuesta que se parezca a la propuesta de Elena White, por lo que consideramos que este artículo corresponde a una investigación casi exploratoria, vista desde la perspectiva de White, es decir no hay fuentes de información sobre el particular. Este trabajo nos permitirá conocer la importancia de este trabajo en beneficio de nuestras instituciones educativas adventistas de incluir las actividades cocurriculares o extracurriculares en el plan de estudios (universidades) y en el plan curricular (nivel básico), la sociedad reflexiona y valora el impacto de estas actividades en la mejora del carácter del estudiante y tendrán un mejor rendimiento académico. Asimismo, indagar si existe una clara diferencia entre las propuestas educativas y la de Elena White respecto a las actividades cocurriculares por lo que nos preguntamos ¿Cuál es la propuesta de Elena de White respecto a las actividades cocurriculares y cómo se relacionan con una propuesta posmoderna? Para responder a esta interrogante compararemos las propuestas de las actividades cocurriculares en Elena White y autores contemporáneos a través de un análisis de las propuestas, características y funciones de las actividades co-curriculares contemporáneas; análisis de las propuestas, características y función de las actividades cocurriculares en Elena White para finalmente contrastarlas y relacionarlas, a partir de un análisis bibliográfico. Los resultados indican que las actividades cocurriculares en Elena White, trasciende las propuestas contemporáneas porque prepara a los estudiantes física, social, espiritual e intelectualmente, en tanto que la propuesta contemporánea considera que las actividades co-curriculares son un apoyo fundamental para los estudiantes y sirven para ampliar sus niveles de conocimiento, investigación y currículo.

\section{Actividades cocurriculares}

Las actividades cocurriculares son programas o acciones intencionales que realizan los estudiantes fuera de su horario de clase, las mismas que sirven para incrementar las competencias a través de experiencias significativas y vividas; así como también moldea el carácter del estudiante (Rasi, 2002). En algunas universidades del Latinoamérica y el mundo están en su plan de estudios, tal como se manifiesta en el siguiente párrafo: “..., el universitario participa con 240 horas en actividades cocurriculares, cuyos objetivos son: promover la reflexión y la vivencia de los valores cristianos, la recreación y la vivencia de la práctica deportiva, la participación en actividades artísticas y culturales, los valores cívicos y la 
participación ciudadana, la educación para la salud, la actividad pastoral y el respeto y amor a la naturaleza" (Flores, 2004)

Para el Ministerio de Educación del Perú, según la Resolución de Secretaria General N ${ }^{\circ}$ 297 (Minedu, 2015) "Las actividades cocurriculares, son una serie de recursos auxiliares para ilustrar y objetivar los temas de enseñanza, con el fin de alcanzar la meta primordial de la escuela, que es la de lograr la educación y la formación integral de los individuos" (Salinas, 1996).

En tanto que, para Salinas (1996), "Las actividades cocurriculares, son una serie de recursos auxiliares para ilustrar y objetivar los temas de enseñanza, con el fin de alcanzar la meta primordial de la escuela, que es la de lograr la educación y la formación integral de los individuos" (Salinas, 1996)

Del mismo modo, (Gallardo, 2007, p. 2) entiende por:

"extracurriculares" a aquellas actividades estructuradas e inestructuradas a las cuales los estudiantes acceden en el contexto universitario, que no son parte de los procesos de instrucción formal de la institución educativa, y que sin embargo influyen en el desarrollo global de los estudiantes, fomentando la adquisición de habilidades cognitivas y sociales en sus participantes.

Asimismo, Castro (2003, p. 37) sostiene que:

las actividades extraescolares o cocurriculares, son aquellas que el personal académico realiza como parte de la formación integral en el contexto educativo, contribuyen a concretizar los aspectos vocacionales de los sujetos, y obedece a un marco constitucional de planear dichas actividades, las cuales puede llevarse a efecto dentro de las instalaciones de la institución, o bien fuera de ellas, con el objetivo de lograr un proceso de enseñanzaaprendizaje que sea efectivo en los estudiantes.

Igualmente, (Lainez, s/a) define las actividades extraescolares como aquellas que están encaminadas a potenciar la apertura del centro educativo a su entorno y a procurar la formación integral del alumnado. Estas actividades siempre se realizarán fuera del horario lectivo, tendrán carácter voluntario para el alumnado y buscarán la implicación activa de toda la comunidad educativa.

Respecto a las actividades cocurriculares en el pensamiento de Elena de White y las de la Iglesia Adventista del Séptimo Día, conciben que la verdadera educación como un proceso de desarrollo armonioso y equilibrado del ser humano en sus aspectos físico, intelectual, social y espiritual. Esto significa que en el proceso educativo no corresponde privilegiar ninguno de estos aspectos en desmedro de otro(s), sino que todos deben ser atendidos por igual. Implica que la racionalidad, individualidad, la salud, el servicio, la cooperación y la continuidad, son 
atendidas desde diferentes frentes y para cumplir los propósitos globales deberían formar parte del currículo como actividades cocurriculares, si hablamos en términos actuales.

\section{Propuesta Educativa "Mundial"}

\section{Propuesta de Actividades Cocurriculares en la India}

En este país, en el Marco Curricular Nacional 2005, (NCF2005), tiene el objetivo de desarrollar competencias individuales de los educandos para que tengan la facultad de tomar decisiones que transformen favorablemente sus propias vidas y su sociedad, desde dos frentes: 1. Educación centrada en el trabajo (NCF2005) estimula así el emprendimiento, la solución de problemas, la observación, la toma de decisiones, la lluvia de ideas y la evaluación de soluciones como competencias que deben ser adquiridas por un estudiante cuyo objetivo sea adaptarse a situaciones laborales cambiantes. Estas competencias permiten el aprendizaje para la vida, que es esencial en los ambientes laborales de la industria, la agricultura y las corporaciones. El método propuesto en todas las materias se centra en proyectos colaborativos, tales como trabajo e investigaciones de campo, indagaciones y gestión de granjas locales que lleven a actividades de aprendizaje que motivan el conocimiento aplicado a una cuestión práctica" (Reimers, 2016); 2. Mente, corazón y manos, promueve los respetos por la dignidad del trabajo y la especialización no formalizada de gente como artesanos y agricultores. El Marco Curricular Nacional (NCF2005), también empodera tanto al estudiante como a la comunidad al introducir como maestros proveedores de recursos especiales a artesanos, carpinteros y agricultores en la educación formal, con lo que estimula los ámbitos cognitivo, afectivo y psicomotor de manera holística" (Reimers, 2016).

\section{Propuesta de actividades cocurriculares de la University of Eastern África}

En el caso de África, gracias a los buenos oficios de un miembro adventista del parlamento, el presidente de Kenya concedió a la iglesia un contrato a largo plazo para establecer una institución postsecundaria en el terreno de una antigua estación agrícola (Greenleaf, 2010). Las ofertas académicas se ampliaron rápidamente hacia campos vocacionales. Los instructores en programas de lechería y agricultura realizaron experimentos en cooperación con las agencias agrícolas de Kenya... En 1990 la institución recibió una donación de medio millón de dólares del gobierno de los Estados Unidos para construir y equipar laboratorios de automecánica y reparación de vehículos y para ofrecer una licenciatura con especialidades en tecnología de automotores y en la enseñanza de mantenimiento de vehículos (Greenleaf, 2010). 


\section{Propuesta de actividades cocurriculares de la Universidad Autónoma de Nueva León,} México

Entre las actividades cocurriculares sugeridas por el documento "Reforma académica en el nivel medio superior", se encuentran: 1) Trabajo cotidiano de consulta en bibliotecas y hemerotecas. Consiste en motivar a los estudiantes para acudir a estos centros a resolver sus dudas, ampliar sus conocimientos y conocer cómo funciona este tipo de instituciones; 2) Asistencia a diferentes actos culturales y artísticos; 3) Visitas a museos y exposiciones diversas; 4) Elaboración de periódicos murales; 5) Participación en concursos variados; 6) Investigación de campo. Dará al estudiante la oportunidad de trabajar vivencialmente con los fenómenos científicos que conforman la materia de estudio y hará sentirle parte importante de ella; 7) Creación de videos-clubes y círculos de lectores; y 7) Talleres de creación. (Nava, 1996).

\section{Propuesta de actividades cocurriculares de la Universidad Monte Morelos- México}

Dentro del Plan de Estudio de las diferentes carreras consideran actividades denominadas "Formación para el servicio" tales como: agricultura y ecología; mantenimiento del hogar; industria; emprendimientos; ejecución de proyectos; arte culinario, mecánica, cosmetología, carpintería, albañilería, fontanería, entre otros.

\section{Propuesta Educativa Peruana}

\section{Nivel Básico}

La propuesta de actividades cocurriculares en el nivel básico (inicial, primaria y secundaria) no se encuentra bien definido por niveles. El Ministerio de Educación ha dispuesto horas de libre disponibilidad, (que podrían utilizarlas para actividades cocurriculares) de acuerdo a niveles; por ejemplo, el nivel Primaria y Secundaria, 2 horas; y algunas actividades para estos niveles dentro de la Estructura Curricular Básica (ECB) a través de proyectos, tal el caso en el área de ciencia y ambiente, en comunicación, personal social, entre otros.

\section{Nivel Universitario}

Algunas universidades presentan actividades extracurriculares, tal es el caso de la Universidad Nacional de Moquegua, que en su reglamento de actividades cocurriculares, aprobado por resolución de comisión organizadora No 0161- 2016- UNAM, de fecha del 26 de julio de 2016, dice: "Los estudiantes que tengan talento deportivo y destaquen en las actividades curriculares serán incluidos en los programas de alta competencia (PRODAC) de las distintas categorías y disciplinas deportivas para representación institucional (programas deportivos) a 
desarrollarse a través de las actividades cocurriculares, futbol, básquet, vóley, atletismo, entre otras natación, futsal, ajedrez, tenis de mesa, frontón, juegos de salón. Los estudiantes que tengan talento artístico y destaquen en las actividades cocurriculares serán incluidos en los servicios culturales para representación institucional elenco de danzas, elenco de música, tuna universitaria, coro universitario, pintura, escultura y teatro" (Laqueticona, 2016)

La Universidad Andina del Cuzco, aprueba su Directiva No10-2016/VRAC-UAC, sobre las actividades extracurriculares a través de la resolución No 306-R-2016-UAC, de fecha 17 de octubre de 2016, dice: Se considera actividades extracurriculares aquellas que el estudiante realiza fuera del aula y que inciden en su formación integral. Son actividades que pueden tratarse de: "Participación en eventos deportivos, dentro o fuera de la Universidad de cualquier disciplina solo (en disciplinas pertinentes) o integrándose dentro de un equipo. En este caso el entrenador o responsable acreditará al estudiante, sus horas de ensayo, así como las de su presentación. Participan en danza, música, teatro, pintura, escultura, recitales de poesía, cine, fórums y otras, participación acreditada por el titular de la actividad con documentos que la demuestran (programa del evento, programa de la exposición, certificado de participación y otros, etc.). Asistencia a seminarios, fórums, mesas redondas, talleres, charlas de investigación, especialidad o desarrollo personal certificados por los organizadores. Integrar y participar en brigadas universitarias o comunitarias de diversa índole, de control, de rescate y otras, participar activamente en voluntariados de diversa índole, simulacros de desastres naturales, simulacros de incendios y otras actividades. Participar en acciones de responsabilidad social desarrolladas por su Escuela Profesional correspondiente" (Cuzco, 2016)

Asimismo, la Universidad Científica del Sur, en su reglamento de estudios de pregrado, en el capítulo VII, sobre el régimen de actividades extracurriculares, artículos 46 y 47 del mes de enero de 2015, dice: "La Universidad fomenta entre los estudiantes la participación en actividades culturales, deportivas y de responsabilidad social a través de la Facultad y otras áreas de la Universidad, con el fin de promover su formación integral. El Decano/Director en concordancia con su plan de estudios podrá proponer el número de créditos para la participación de sus estudiantes en las actividades extracurriculares. La evaluación de la participación de los estudiantes, los hará acreedores de una nota, la misma que deberá ser establecida de acuerdo al reglamento correspondiente, aprobado por el Vicerrectorado Académico" (Universidad Científica del Sur, 2015). 
La Universidad Peruana Unión es única en el país en cuanto a sus actividades extracurriculares, que en su reglamento del estudiante unionista, aprobado por resolución N²268-2016/UPeU-CU, de fecha 24 de noviembre de 2016 y en el capítulo VIII, artículos 98, 99 y 100 dice lo siguiente:

Los grupos organizados pueden realizar retiros y campamentos, salidas de estudio, asistencia a eventos académicos y excursiones en fechas debidamente programadas y de acuerdo con los lineamientos generales de la UPeU, requiriendo la autorización previa de la instancia a la que dependan y ratificada por el Consejo Universitario con veintiún (21) días de anticipación. El Consejo Universitario emite la autorización conforme a las normas establecidas para dicho efecto. Las salidas de las diversas áreas son aprobadas por el Consejo Universitario. Los retiros, campamentos, giras, excursiones, paseos, cenas de gala, encuentros de grupos mixtos se desarrollan con presencia de los preceptores capellanes o docentes o consejeros autorizados y bajo la responsabilidad de las autoridades de la EP o facultad o EPG. La exhibición de películas, vídeos u otros espectáculos artísticos deben reflejar la filosofía de la UPeU. Los decanos o la DG de Bienestar Universitario son los responsables del cumplimiento de esta norma, en coordinación con el preceptor o capellán o docente o consejero o responsable del área o de la EP o EPG (Universidad Peruana Unión, 2016, p. 34)

Las actividades extra-clase constituyen una excelente oportunidad para la actuación educativa del profesor, además de proporcionar al educando campo de experiencia e inspiración en las actividades vocacionales. Se trata, por lo tanto, de actividad útil a los profesos, que podrán aprovechar los recursos de su disciplina para motivarla y difundirla en la escuela (Pereira, 1971) "Las actividades extraescolares, por su parte, tienen como característica principal la participación voluntaria o autodeterminada y son actividades que se realizan por fuera del horario escolar" (Calero, 2016, p. 103)

\section{Actividades Cocurriculares en Elena White: Propuestas}

Partimos desde los presupuestos básicos respecto a la educación, E. de White. Considera que la educación correcta responde a: "Los niños y los jóvenes necesitan una buena educación, esta educación pueda servir en la vida cotidiana de los niños y jóvenes. Sin descuidar la moral pensando que la parte intelectual es todo. Asimismo, es necesario, instruir, cultivar, pulir y refinar a los jóvenes y los niños, debiera ser la preocupación principal de padres y maestros [...] a veces los maestros y los padres confían mucho en la parte teórica pensando que lo hace todo, pero en realidad es incompleto, porque faltaría las actividades extracurriculares (talleres) y la parte moral" (CM 83.1)

En este marco se dirige a las instituciones educativas y les señala: "Sería bueno que, de ser posible, hubiera en relación con nuestros colegios, tierras para el cultivo y talleres, bajo la dirección de hombres competentes para instruir a los estudiantes en los diversos aspectos del trabajo manual". (CM. 86.1) 
Consideramos que este es uno de los párrafos en los que podríamos sustentar, en E White, los programas cocurriculares y su razón de ser: [...] todos los estudiantes deberían extraer conocimientos de las actividades extracurriculares o cocurriculares para ganarse la vida. El aprendizaje de las actividades extracurriculares (talleres) es una garantía contra las vicisitudes de la vida, la influencia será notorio de estas actividades extracurriculares en el desarrollo físico, mental y espiritual. A pesar que un niño no nace con esas habilidades de aprender de las actividades extracurriculares como un sustento posterior, hay que enseñarles a trabajar por lo menos. Para tener buena salud y saludable es importante considera el ejercicio físico de diferentes formas haciendo la parte práctica de las actividades extracurriculares y la disciplina del trabajo bien regulado, no es menos esencial para obtener un espíritu fuerte y activo que para adquirir un carácter noble. (CM 292.2)

Desde esta concepción entendemos que existe una educación que va más allá de un simple conocimiento. La continuación de un determinado curso, no significa una verdadera educación, sino están las actividades extracurriculares. Significa más que una preparación para la vida actual. La educación abarca durante la existencia del hombre. Durante este período es necesario desarrollar las facultades físicas, mentales, y espirituales en forma armoniosa. Prepara al estudiante para el gozo de servir en este mundo, y para un gozo superior proporcionado por un servicio más amplio en el mundo venidero" (Ed.13).

En cuanto a la propuesta del desarrollo armonioso de las facultades en el ser humano, E. White, en el ángulo del desarrollo espiritual considera que: "Todo verdadero conocimiento y desarrollo tienen su origen en el conocimiento de Dios. Doquiera nos dirijamos: al dominio físico, mental y espiritual [...] Cualquier ramo de investigación que emprendamos, con el sincero propósito de llegar a la verdad, nos pone en contacto con la Inteligencia poderosa e invisible que obra en todas las cosas y por medio de ellas. La mente del hombre se pone en comunión con la mente de Dios; lo finito, con lo infinito" (Ed. 98 14.2).

Desde esta afirmación notamos una diferencia marcada con las propuestas curriculares modernas. De ahí que la autora considera que educación y salvación son una sola. Al realizar estas actividades extracurriculares deberían reflejar la imagen de Dios para la redención y la restauración del hombre, devolverlo a la perfección con que había sido creado, promover el desarrollo de las facultades del hombre, a fin de que se llevara a cabo el propósito divino de su creación. Este es el objetivo de la educción, el gran propósito de la vida (ED. 15.2). 
Si desmembramos el desarrollo integral al que se refiere la autora, dando un orden en el desarrollo físico, mental, espiritual y cómo éstos se logran dentro de las actividades cocurriculares, extraemos los siguientes textos. "El crecimiento intelectual y espiritual debe ser tan marco como el desarrollo de las fuerzas físicas. Los jóvenes deberían sentir la necesidad de ser fuertes y competentes, tanto intelectual como espiritual. (COES 94.2).

Los párrafos siguientes integran el todo del ser humano:

Los buenos hábitos de comer, dormir, estudiar y hacer ejercicios, agradan a Dios, asimismo estos vienen a ser el mayor ejemplo para la sociedad actual. Al obtener los estudiantes la verdadera educación por lo tanto ellos serán una bendición para el mundo, esto ayudará a alcanzar los objetivos trazados de la iglesia y de la sociedad venidera. Muchas veces hay estudiantes solo se dedican al estudio teórico y se descuidan la parte de la alimentación, pocas horas duermen y no hacen ejercicios con regularidad o simplemente muchas veces los estudiantes consumen alimentos de mala calidad o inadecuadas, todo esto no agrada a Dios (ED 380.2).

Tener en consideración de aquellos alimentos que se relaci0nan con la parte cognitiva del estudiante, de cómo está siendo atendido hasta el momento [..] es importante mostrar a los estudiantes el valor nutricional de los alimentos [...] considerar no solo las propiedades de los alimentos, sino también su adaptación a quien los consume [...] no se debe olvidar de recibir los alimentos en el horario ya establecido, caso contrario traerá consecuencias fatales [...] Al mismo tiempo, la adquisición del dominio propio será una condición muy importante, porque sin esto no podrá tomar decisiones ni mucho menos cumplir los deberes de la vida cotidiana (ED 186, 187).

La combinación de los alimentos es muy importante para el desarrollo intelectual y no olvidarse de los cereales, frutas diversas, nueces y los vegetales; estos alimentos sobre todo constituyen en la preparación mental y físico (ED 98, 204.3).

En estas pequeñas citas podemos desprender varias realidades que las instituciones educativas no podrían desarrollarlas a menos que tengan, dentro de su currículo actividades extra curriculares que le permitan ese desarrollo armonioso de las facultades. Uno de los objetivos de la educación es lograr la disciplina mental, con un desarrollo simétrico de todas las facultades o aptitudes de la mente. "La educación disciplinará la mente, desarrollará sus facultades, y las orientará en forma inteligente, para que podamos ser útiles en promover la gloria de Dios" (Cadwallader, 2011). 
Otro de los objetivos de la educación adventista se encuentra en el área del servicio. Por ejemplo, la educación debiera: capacitar a los niños y a los jóvenes para llevar responsabilidades y preparar a cada uno para realizar una obra noble para Dios y ser una bendición para la humanidad.

"El verdadero objetivo de la educación debería considerarse cuidadosamente. Dios...de cada uno de nosotros que cultivemos nuestras facultades y logremos la mayor capacitación posible a fin de ser útiles, para que podamos realizar una obra noble para Dios y ser una bendición para la humanidad" (Rasi, 2002).

Los temas de desarrollo del carácter, la función de la iglesia cristiana, estudio de la Biblia, historia, literatura y ciencia, fueron revisados y analizados por la Elena White. Desde su perspectiva todos estos temas tenían un solo objetivos era de reflejar la presencia de Dios y la conversión, asimismo era importante la preparación de jóvenes con disposición de servicio a la humanidad actual (Knight, 2015).

Las características distintivas de la educación adventista- basadas en la Biblia y en los escritos de Elena White- destacan el propósito redentor de la verdadera educación: recuperar la imagen del hombre para que pueda reflejar la imagen de Dios. Por intermedio de la educación se puede reestablecer la comunicación original con Dios; relacionando de manera tridimensional: el hogar, la educación y la iglesia de esta forma los agentes divinos intervinieran en los educandos y de esa forma pueden ser ciudadanos responsables en este mundo y lleguen a ser ciudadanos del mundo venidero (Rasi, 2002).

Por otro lado, además del desarrollo físico y mental, la propuesta de Elena White sobre las actividades cocurriculares, trasciende al considerar que debería preparase a los estudiantes para ser útiles en la vida, forma el carácter, y enriquecer la vida profesional de los estudiantes a través de estas actividades. El estudiante debe estar preparado para ser útil en la vida y servir en forma general y cada día a la humanidad, y también para servir en la iglesia y en el campo misionero; entendiéndose en cualquier lugar de la tierra donde haya necesidad de colaborar con el propósito mayor de la educación.

Para librarse de la pereza estudiantil es bueno asignar trabajos de acuerdo a su capacidad y edad, es cuán importante tener en cuenta en plena formación del carácter del estudiante. Han de familiarizarse con los deberes de la vida diaria. Se les debería enseñar a cumplir bien y esmeradamente sus deberes domésticos, con el menor ruido y confusión posible (White, 2004). "Más que ningún otro agente, el servir por amor a Cristo en las cosas pequeñas de la vida diaria 
tiene poder para formar el carácter y para dirigir la vida por el camino del servicio abnegado. Despertar este espíritu, fomentarlo y encauzarlo debidamente es la obra de padres y maestros" (Cadwallader, 2011). "Por ello debería ocupar un lugar importante en el plan de estudios, porque este tipo de actividades cocurriculares es de mayor provecho para los estudiantes que los juegos y los deportes" (Cadwallader, 2011).

\section{Trabajo manual}

La responsabilidad del maestro cristiano en la vida del estudiante es muy significativa, de él depende el desarrollo la instrucción, el inculcarles valores, enseñarles a ser útiles en la vida, es decir el trabajo no solo es estar impartiendo enseñando en el aula, es prepararlo de manera integral para este mundo y el venidero

“Coopere cada maestro cordialmente con un grupo de alumnos, trabajando con ellos y enseñándoles a trabajar. Mientras los maestros hagan esto, adquirirán valiosa experiencia. $\mathrm{Su}$ corazón se unirá con el de los alumnos, y eso preparará el terreno para que su enseñanza tenga éxito" (CM, 195.2) Nuestros maestros no deberían pensar que su trabajo terminó con impartir instrucción de los libros. Debieran dedicar varias horas cada día a trabajar con los alumnos en algún ramo de la preparación manual. En ningún caso se la debiera descuidar. (CM, 202.2).

Sería bueno que, de ser posible, hubiera en relación con nuestros colegios, tierras para el cultivo y talleres, bajo la dirección de hombres competentes para instruir a los estudiantes en los diversos aspectos del trabajo manual. Mucho se pierde por descuidar la unión del trabajo físico con el mental. (CM 86.1) “... era costumbre de los judíos, tanto de las clases más ricas como de las más pobres, enseñar a sus hijas e hijos algún oficio útil, para que si se presentaban circunstancias adversas, no tuviesen que depender de otros, sino que pudieran proveer a sus propias necesidades... Esto era considerado como parte indispensable de su educación" (CM, 292.1) "Los alumnos deben salir de nuestras escuelas dotados de eficiencia cabal, a fin de que cuando dependan de sus propios recursos, tengan conocimientos que puedan usar, útiles para tener éxito en la vida. Es esencial el estudio diligente, pero también lo es el trabajo arduo y laborioso" (CM, 493.4)

Cabe resaltar también, opiniones de las personas de sociedades modernas y posmodernas que han tratado de fundamentar que los hombres que hacen trabajo manual no deben ser reconocidos como caballeros, tal como se presenta a continuación: El trabajo manual es una bendición de Dios para su pueblo, pero la sociedad ve a la agricultura como una actividad no digna, no de caballeros; la actividad digna es aquella que da riquezas materiales, dejando a 
un lado la riqueza interior, la que modela el carácter del niño, haciéndolo un ciudadano para la patria celestial. El trabajo manual dignifica al hombre y sobre todo a los estudiantes en pleno desarrollo intelectual, pero muchos lo consideran algo degradante. El pensamiento y la opinión del hombre muchas veces prohíbe el trabajo manual, en vez que preparen a sus hijos a ser responsables y a trabajar desde pequeño de acuerdo a su capacidad (White, 1971).

La educación manual merece más atención de la que se le ha prestado. La cultura mental y moral no está mal, pero es importante también proporcionar la educación manual para desarrollar la parte física e industrial. Se debe enseñar los siguientes oficios: agricultura, trabajos manuales, economía doméstica, arte culinario, costura, confección de ropa higiénica, tratamientos a enfermos y otras cosas parecidas. Se debe disponer de jardines, talleres y salas de tratamientos y la dirección del trabajo, en todas loa ramas, tiene que estar a cargo de instructores expertos (Cadwallader, 2011).

\section{La agricultura}

Vivimos en una sociedad posmoderna que se da más prioridad a la tecnología, dejándose de lado el trabajo en los campos agrícolas, tal como lo manifiesta la sierva de Dios: "Los padres deberían preparar a sus hijos para que se ocupen con ellos en sus oficios y empleos. Los agricultores no deberían pensar que la agricultura es una ocupación que no es suficientemente elevada para sus hijos. La agricultura debería progresar mediante el conocimiento científico". (White, 2015).

"El ejercicio en tareas agrícolas y en las diversas ramas del trabajo son una salvaguardia maravillosa contra el esfuerzo indebido del cerebro. El trabajo diligente resguarda a muchos, jóvenes y viejos, de las trampas de aquel que "encuentra algún mal para que hagan las manos ociosas" (White, 2014).

Desde la fundación del mundo, Dios sabía que el ser humano iba a tener dificultades en su vida cotidiana, porque habría un conflicto cósmico entre el bien y el mal, por ello en su sabiduría el creador dio al hombre actividades que se mantuviera ocupado para no ceder a las tentaciones. Las consecuencias de la ociosidad son: el vicio, el crimen y la extrema pobreza. Si el hombre estuviera con manos ocupados la sociedad actual sería diferente, es importante la ocupación y cumplir con las tareas y deberes diarios. El trabajo diligente los guarda de muchas de las trampas de aquel que "halla siempre alguna mala ocupación para las manos ociosas (White, 1971).

Las actividades cocurriculares en el pueblo Israel eran consideradas como un deber tanto para hombres como para las mujeres, y era un honor trabajar en las industrias, que les servía para su sostenimiento diario. En la época de los israelitas el mayor deber era la preparación 
industrial. En ese entonces el padre ya sabía inculcar un oficio útil a su hijo. Había hombres encargados especialmente para adiestrar en actividades industriales, porque ellos consideran como una actividad importante, asimismo clasificaban a las damas y a los hombres de acuerdo a sus habilidades. Se enseñaban diversas industrias en las escuelas de los profetas, y muchos de los estudiantes se sostenían por el trabajo manual" (Ibídem, 1971). "Los padres deberían inventar nuevas formas y medios para mantener a sus hijos ocupados en algo útil. Que los niños reciban pequeños lotes de tierra para cultivarlos, con el fin de que tengan algo que ofrecer como una ofrenda voluntaria" (White, 2015).

\section{La granja y/o finca}

La granja o finca representaba en bien inmueble por excelencia y estaba constituida por extensas áreas de terreno. Ha tenido gran importancia desde muchos siglos antes de Cristo por su auge económico en las épocas previas a la industrialización, siendo el símbolo de riqueza y prosperidad.

Los jóvenes que asistan a nuestras escuelas necesitarán todo el terreno cercano. Han de poner en él plantas de adorno y árboles frutales, y cultivar la huerta. La finca de la escuela ha de considerarse como un libro de texto de la naturaleza, del cual los maestros saquen lecciones prácticas. Se ha de enseñar a nuestros alumnos que Cristo, quien creó el mundo y todas las cosas que hay en él y la luz de cada cosa viviente (White, 2008).

El plan de Dios fue que los seres humanos tengan en sus hogares áreas de terreno con fines decorativos y ornamentales, como lo fue al principio cuando Dios creó a la primera pareja. Los niños y los jóvenes aprecian un método sencillo, las mejores lecciones se aprenden en aire libre o relacionando con la naturaleza, estas lecciones ayudarán de gran manera a los niños y los jóvenes que tienen problemas de retención inmediata. La naturaleza trae felicidad y salud; y las impresiones hechas no se desvanecerán de su mente, porque quedarán asociadas con objetos que están continuamente delante de sus ojos (White, 1971).

\section{Tareas domésticas}

Las tareas domésticas es una actividad de todos los que viven en una casa, es decir se deberá cumplir según a cabalidad de acuerdo a las edades de sus miembros. Beneficia:

Los trabajos domésticos ayudarán más a las niñas. La labor física no impedirá que cultiven el intelecto; lejos de ello. Uno de las ventajas de la labor físico es equilibrar el estrés mental de las personas. El trabajo recaerá sobre los músculos y aliviará el cerebro cansado... el cerebro sano requiere un cuerpo sano. La continuación de los deberes caseros es la sanidad física y el conocimiento práctico, estos nunca impedirán al desarrollo intelectual de ser humano 
(White, 1971) "Cuando una niña tiene nueve o diez años de edad debería pedírsele que se haga cargo en forma regular de las tareas domésticas, en la medida de su capacidad, y que se responsabilice por la forma en que realiza su tarea" (Cadwallader, 2011).

\section{La cocina}

Se dice que cocinar es un arte. No todos en esta vida se tiene el buen arte de cocinar, pero en la actualidad existe escuelas especializadas en el arte culinario. La recomendación para cada hogar es que todos hombres y mujeres deberían practicar el arte culinario. "Es muy esencial que el arte culinario sea considerado una de las ramas más importantes de la educación. Los hombres y las mujeres deben ser enseñados cómo preparar los alimentos...Esta rama de la educación debe ser impartida en cada escuela establecida. Los estudiantes deben ser enseñados con paciencia como cocinar. El conocimiento de la reforma en el régimen alimenticio...es una parte esencial de la educación que ha de darse en nuestras escuelas” (CSRA, 316).

En el área de arte culinario las damas necesitan un experto para recibir las lecciones, asimismo los jóvenes necesitan un especialista en el rubro de corte confección y los demás deberes prácticos de la vida, también estos oficios requieren un ambiente bien equipado para que puedan cumplir con el desarrollo de sus facultades mentales (White, 1971).

La realidad de algunas mujeres casadas en la actualidad es triste de no saber cocinar, ni hacer pan, cortar una pieza de telas, hacer ropas, estos conocimientos prácticos son muy importantes en el hogar. Algunas señoritas piensan que es suficiente leer, adquirir conocimientos teóricos o tocar un instrumento bastaría todo, pero en realidad no es lo suficiente (White, 1971).

Generalmente algunas señoritas en la actualidad se excusan al momento de elegir el trabajo doméstico, porque se consideran como sirvientas. Pero en realidad no lo es, sino lo contrario. Las mamás tienen un deber muy importante de motivarlas a que puedan aprender desde pequeñas los trabajos domésticos en la casa. El mejor trabajo en el hogar es saber cocinar, aunque otros trabajos vendrían después como ser costurera, ser dactilógrafa o la maestra de música. Sin alimento el hombre no puede vivir es más saber cómo llamar la atención con las comidas en la mesa, los pequeños detalles requiere inteligencia y experiencia (White, 1971).

"En todas nuestras escuelas debe haber quienes estén preparados para enseñar el arte culinario. Deben dictarse clases para impartir instrucción en esta materia. Los que se están 
preparando para servir sufren una gran pérdida cuando no adquieren el conocimiento acerca de cómo preparar los alimentos para que sean a vez sanos y apetitosos" (White, 2008).

\section{El Colportaje}

El colportaje es un ministerio sagrado y de entrega completamente a Dios; es una actividad que no todos lo hemos experimentado. Esta actividad ha agrupado a muchos jóvenes en la venta de libros, revistas, entre otros; con la finalidad de difundir el mensaje de la segunda venida de Cristo y también un medio para sufragar gastos de estudios. "El Señor ha instituido un plan por medio del cual un buen número de alumnos de nuestras escuelas pueden aprender lecciones prácticas, que les asegurarán el éxito en su carrera. Les da ocasión de vender libros preciosos, consagrados al adelantamiento de nuestra obra de educación y de salud. El mismo hecho de vender esos libros presentará a los jóvenes muchas incidencias que los prepararán para resolver los problemas que los esperan en las regiones lejanas. Al vender estos libros durante su vida escolar, muchos pueden aprender a acercarse a la gente de una manera cortés y a discurrir con tacto sobre los diferentes puntos de la verdad presente" (White, 2004).

El negocio necesita trazar metas claras y juiciosas, como la venta de los libros, que estos materiales puedan edificar el hogar, al mismo tiempo sería una ayuda financiera para los estudiantes para que puedan continuar sus estudios universitarios lo más importante de este colportaje es ganar la experiencia, porque esto servirá al estudiante a prepararse como obrero misionero para llevar la palabra de Dios sin miedo (White, 2004).

\section{Características de las actividades cocurriculares}

\section{Los talleres}

Los talleres son asociaciones libres para el logro de fines comunes; proporcionan un sentimiento de pertenencia, de libertad responsable y la posibilidad de participar y recrearse, favoreciendo el ejercicio de la creatividad. Un taller creativo ofrece condiciones para ejercer no sólo habilidades, sino que en él probamos hasta dónde podemos proyectarnos; es un medio de desarrollo y/o recuperación de la capacidad expresiva, donde buscamos una zona libre de presiones de un sistema que nos exige acatamiento y productividad, donde somos protagonistas de nuestro quehacer y espectadores en todo lo demás (Salinas, 1996)

El propósito real de la disciplina en el taller o en el aula es lograr que cada individuo dedique todo el tiempo aprovechable a hacer firmes progresos en su formación profesional, al mismo tiempo que trabaja como parte del grupo, sin sufrir la interferencia de otros y sin 
interferir él mismo el progreso de los demás. De esta manera, la disciplina establecida por el maestro puede eventualmente convertirse en auto disciplina de cada individuo. Solamente cuando este tipo de disciplina se generaliza entre los de una clase puede desarrollarse entre ellos y el maestro una relación de amistad, de franco y respeto mutuo, en una atmósfera de tranquilidad, y solo así prevalece una plena actividad en el taller (Delmar, 1969)

El maestro de taller puede esperar que gran parte de este entrenamiento ya haya sido efectivamente realizado por los padres y por maestros anteriores de sus alumnos antes de que se inscribieran en su clase. Un taller se caracteriza, ante todo, por la cohesión, es decir, la adhesión afectiva y cognitiva de sus miembros, así como por la integración determinada en base a la flexibilidad del grupo. Otro aspecto importante es la interacción que se da a partir del reconocimiento de los sujetos que lo constituyen y de las necesidades, inquietudes y aspiraciones de los mismos (Salinas, 1996)

\section{Preparación docente}

La discusión es uno de los métodos que requieren cuidadosa preparación por el maestro antes de ser utilizado y un adecuado control del maestro cuando se está desarrollando. No es importante que el maestro, particularmente si es inexperto, comience una clase con discusión abierta súbitamente o como simple recurso improvisado para emplear un tiempo disponible (Delmar, 1969).

Los maestros deben aceptar siempre de buen grado la responsabilidad de proyectar otras tareas escolares para promover una mayor eficiencia del programa general de la escuela. Ninguna buena escuela puede funcionar sin la ayuda del maestro en la operación de locales, orientación de los estudiantes, clubes, publicaciones, actividades atléticas y otra extracurriculares (Delmar, 1969).

\section{Beneficios}

Las sociedades en el mundo y las escuelas en particular están promoviendo que en sus currículos o planes de estudio se integren actividades cocurriculares como un valor agregado para tener una formación integral y ser útiles en la vida: Las Escuelas están desarrollando también las competencias para el siglo XXI en los alumnos mediante una amplia gama de actividades cocurriculares que sirven como plataformas naturales para el desarrollo de habilidades y valores. Siempre han existido estas actividades en las escuelas y son plataformas auténticas para el desarrollo de las competencias del siglo XXI al brindar contextos perfectos 
para el aprendizaje y la asunción de valores morales, la adquisición y práctica de las habilidades transversales o suaves (la capacidad de comunicarse y relacionarse con los demás la denominada "inteligencia emocional": en suma, las aptitudes interpersonales) y la integración social de los niños de entornos y grupos étnicos diferentes (Reimers, 2016).

Dentro de las actividades cocurriculares, las instituciones de nivel superior se preocupan por entregar a través de un plan o programa de actividades competencias que no desarrolla la capacidad intelectual del estudiante, por ello se sugiere que dichas actividades deben estar encaminadas a desarrollar competencias de siglo XXI, tales como actividades de innovación tecnológica, creatividad, entre otros; y prepararlo al estudiante para ser útil en la vida en el siglo XXI.

Sin embargo, ante las actividades cocurriculares se limitan al aprendizaje de competencias adicionales en deportes y arte y no se enfocan en las competencias del siglo XXI. Al fin de utilizarlas como plataforma para enseñar las competencias necesarias en el presente siglo para integrar con éxito esas competencias a las actividades cocurriculares, se adoptó un planteamiento de tres flancos. Primero, se presenta la necesidad de crear la cultura deseada que apoyara la reflexión del alumno y del maestro y fomentara la excelencia personal. Segundo, los directores escolares eran los motores clave necesarios para crear una visión compartida de las actividades cocurriculares en sus escuelas; se debía incrementar la estructura de apoyo de cada actividad cocurricular e instaurar un desarrollo y una preparación profesional más intencionados para los maestros que manejaban estas actividades. Tercero, era necesario identificar las oportunidades para la integración de las competencias para el siglo XXI a las actividades cocurriculares..." (Reimers, 2016).

A través de las actividades cocurriculares, los estudiantes comparten experiencias inolvidables y que éstas le servirán para ser útiles en su vida diaria: "Los conocimientos se aprenden sólo cuando se experimentan personalmente; de ahí que las actividades cocurriculares tengan su principal punto de apoyo en el principio psicológico, de acuerdo al cual el individuo tiene un mejor concepto de las cosas que vive, que de aquellas acerca de las cuales sólo ha leído u oído explicar” (Salinas, 1996).

Las actividades cocurriculares son diferentes, puesto que cada estudiante podrá acceder de acuerdo a las habilidades y destrezas. "Un aspecto digno de destacar es el de la interdisciplinariedad que las actividades cocurriculares posibilitan, ya que el alumno que descubre su poder de creatividad y su capacidad de manejo del lenguaje verá una puerta abierta 
para sus potencialidades en las demás áreas que constituyen el currículo escolar" (Salinas, 1996).

\section{Conclusiones}

Después de haber realizado un breve estudio de los libros de Elena de White y otros autores contemporáneos que habla sobre el tema en particular. Los resultados indican que las actividades cocurriculares en Elena White, trascienden las propuestas contemporáneas porque prepara a los estudiantes física, social, espiritual e intelectualmente, asimismo moldea el carácter, en tanto que la propuesta contemporánea considera que las “actividades cocurriculares son un apoyo fundamental para los estudiantes y sirven para ampliar sus niveles de conocimiento, investigación y currículo" (Castro, 2003, p. 75).

Nuestra universidad cumple con algunas de las actividades cocurriculares expuestas en el documento, pero resaltamos algunas de ellas: Los diferentes talleres denominados "Educación para la vida", que prepara al estudiante para ser algo en la vida, moldea el carácter y lo prepara profesionalmente; asimismo también se practica la agricultura y la horticultura

\section{Referencias}

Académica, S. (1993). Proyecto de Reforma Académica en el Nivel medio Superior. San Nicolás de la Garza: Unversidad Autónoma de Nuena León..

Cadwallader, E. M. (2011). Principios de la Educación Adventista en el pensamiento de Elena de White. Lima: Universidad Peruana Unión.

Calero, A. D. (2016). Actividades Extracurriculares durante la Adolescencia: características que facilitan las experiencias óptimas. Individuo y sociedad, 103-110.

Castro Rubio, L. (2003). El Papel que Juega la Formación Cocurricular en el Incremento de la Calidad Académica de los Estudiantes de la Licenciatura en Ciencias de la Educación de la UJAT. México: Tesis.

Cuzco, U. A. (2016). Directiva de las Actividades Cocurriculares de la Universidad Andina del Cuzco. Cuzco.

Educación, M. D. (26 de Febrero de 2015). Resolución de Secretaria General. Lima, Lima, Perú.

Flores Torres, O. (2004). Universidad de Monterrey. Historia y Desafíos, 1968- 2004. Mexico: Editorial Trillas S.A. 
Forero Mendoza, I. X. (2009). Actividades Extracurriculares un Camino Eficaz para Fortalecer en Conocimiento en la Práctica Universitaria. Laberinto, Vol 9, № 1,7-9.

Gallardo, G. (2007). Actividades Extracucurriculares en la Formación Universitaria. Santiago: Observatorio de Juventud Universitaria.

Greenleaf, F. (2010). Historia de la Educación Adventista : Una Visión General. Argentina: Asociación Casa Editora Sudamericana.

Knight, G. (2015). Los Objetivos de la educación Adventista. Revista de Educación Adventista, $1-4$.

Laqueticona Tintaya, Y. G. (2016). Reglamento de Actividades Cocurriculares de la Universidad Nacional de Moquegua. Moquegua: Universidad Nacional de Moquegua.

Laínez, B., \& y Otros. (s/a). Importancia de las Actividades Extraescolares en la educación Secundaria Obligatoria.

Leighbody, G. (1969). Métodos de enseñanza en el taller. México: Editorial Reverte.

Oros Lenguo, M. (2007). Factores que Interfieren en la Participación de los alumnos de Preparatoria en Actividades cocurriculares. Querétaro.

Palacios López, A. I. (2012). Las Actividades Extracurriculares y el Mejoramiento Cualitativo de la Educación. El Milagro: Universidad Estatal de Milagro

Pozón López, J. R. (2014). Los estudiantes Universitarios ante las Actividades Extracurriculares. Andaluza de Ciencias Sociales, 137-150, N No DOI: http://dx.doi.org/10.12795/anduli.2014.i13.08.

Rasi, H. (2002). Declaración sobre la Filosofía Adventista de la Educación. Revista de Educación Adventista, 15-18.

Reimers, F., \& Chung, C. (2016). Enseñanza y aprendizaje en el siglo XXI : Metas, políticas educativas y currículo en seis países. México: Fondo de Cultura Económica.

Salinas, E., \& De Nava, M. (1996). Una Aternativa Didáctica : Las Actividades Cocurriculares en la Materia de Español en Educación Media Superior. Monterrey: Tesis.

Sur, U. C. (2015). Reglamento de estudios Pregrado. Lima.

Torres, O. F. (2004). Universidad de Monterrey. Historia y Desafíos, 1968-2004. México:Editorial Trillas S.A:

Unidas, S. B. (2007). Santa Biblia. Corea: Reina Valera

Unión, U. P. (2016). Reglamento del estudiante Unionista. Lima. 
White, E. G. (2015). Conducción del Niño. Bueños Aires: Asociación Casa Editorial Sudamericana.

White, E. G. (2014). Mente, Caracter y Personalidad. Tomo I. Buenos Aires: Asociación Casa Editorial Sudamericana.

White, E. G. (2008). La Educación Cristiana. Buenos Aires: Asociación Casa Editorial Sudamericana.

White, E. G. (2008). La Educación. Buenos Aires: Asociación Casa Editora Sudamericana.

White, E. G. (2004). Joyas de los Testimonios. Tomo 2. Buenos Aires: CasaEditorial Sudamericana.

White, E. G. (2004). Joyas de los Testimonios. Tomo 3. Buenos Aires: Casa Editorial Sudamericana.

White, E. G. (1971). Consejos para Maestros Padres y Alumnos acerca de la Educación Cristiana. Estados Unidos de Norteamérica: Publicaciones Internacionales

White, E. G. (1975). Consejos sobre el régimen Alimenticio. Estados Unidos de Norteamerica. Editorial Elena G. de White. 